\title{
Introducing Precautionary Behavior by Temporal Diversion of Voter Attention from Casting to Verifying their Vote
}

\author{
Jurlind Budurushi, Marcel Woide and Melanie Volkamer \\ Technische Universität Darmstadt / CASED \\ Darmstadt, Germany \\ Email: name.surname@ cased.de
}

\begin{abstract}
Most electronic voting systems in use today provide printouts so called voter verifiable paper audit trails (VVPATs). Voters are supposed to verify these before they put them into the ballot box in order to detect election fraud. A number of studies have shown that voters are unlikely to do so when using current systems. Thus, it is very likely that malicious electronic voting systems print the wrong candidates without being detected. We introduce precautionary behavior by providing voters with "just in time" instructions while ensuring that these instructions cannot be manipulated by a malicious electronic voting system. Our approach is evaluated in a user study, showing a highly significant increase in the number of voters that verify, as they found the manipulation we introduced in the printouts for the study.
\end{abstract}

\section{INTRODUCTION}

Electronic voting systems have been introduced in the second half of the 20th century, offering the promise of making the tallying process faster and less error prone. As technology advanced, direct electronic voting systems (DREs) were introduced to support visually impaired voters and to prevent unintentionally invalid cast votes. DREs enable vote casting on a computer that stores the accumulated votes and outputs them at the end of the election day.

Despite the advantages, their security and trustworthiness is controversial. Manipulations are very difficult to detect, if at all. As such, the Netherlands and Germany (among others) have stopped using them. Many states in the U.S. now require that DREs provide human-readable printouts (so called voter verifiable paper audit trails) to address this problem. These printouts reflect voter intent and are deposited into the ballot box either automatically by the DRE (used by Avante and Diebold) or manually by the voter [1], [2], [3] and [4]. They enable election officials to audit the tallying, i.e. depending on the electoral regulation of the state, in more or less polling stations the printouts are manually tallied and the results

Permission to freely reproduce all or part of this paper for noncommercial purposes is granted provided that copies bear this notice and the full citation on the first page. Reproduction for commercial purposes is strictly prohibited without the prior written consent of the Internet Society, the first-named author (for reproduction of an entire paper only), and the author's employer if the paper was prepared within the scope of employment.

USEC '14, 23 February 2014, San Diego, CA, USA

Copyright 2014 Internet Society, ISBN 1-891562-37-1

http://dx.doi.org/10.14722/usec.2014.23037 are compared with the corresponding results output of the electronic voting system.

However, the printouts only enable fraud detection if voters actually verify their printout, before these are deposited into the ballot box. Therefore, the larger the number of voters that verify the more likely it is that fraud is detected. Unfortunately, several research studies show that voters are unlikely to verify their printout when using current systems, independent of including instructions in the election material for verifying printouts [5], [6] and [7]. Thus, there is an indispensable need to encourage precautionary behavior when relying on electronic voting systems.

The goal of our research is to introduce precautionary behavior by temporarily diverting voter attention from casting to verifying their printout. We focus on those systems in which voters manually deposit printouts into the ballot box outside the voting booth. Our idea is to divert voter attention by confronting them with visual and short instructions in the appropriate position at the appropriate time. Furthermore, we consider malicious electronic voting systems that would display a correct review screen to the voters, but manipulate the printouts. By considering malicious voting systems, we are restricted regarding adequate solutions because displaying the instructions to verify by the DRE might be promising from a usability perspective but is inadequate from a security perspective: a malicious DRE would not only manipulate printouts, but may also display incorrect or no instructions to verify. Another challenge is diverting voter attention from verifying back to the next step in the vote casting process, namely folding the printout (in cases where no manipulations occur) before leaving the voting booth to ensure vote secrecy.

In order to address both challenges we propose the use of printouts with pre-printed verification instructions. Once printed, the voter first sees the instructions (to verify and fold) and then needs to turn the printout, in order to verify its content. We evaluated this approach in a user study, comparing it to integration of the same instructions in the election material. The focus of the evaluation lies on integrity, i.e. detecting manipulated printouts. The results of our study show that integrating pre-printed instructions on the printout significantly increases the number of voters verifying and finding the manipulation we introduced in the printout. Furthermore, the instructions seem not to prevent voters from folding the printout. 
This work is structured as follows: Section II is dedicated to related usability research on electronic voting. In Section III the general concepts and the functionality of the addressed electronic voting systems are introduced. Afterwards, in Section IV, we describe the methodology for developing an adequate stimulus to temporarily divert voter attention from their main task (casting the printout) to verifying their printout and back to folding the printout. The study design to evaluate the stimulus effectiveness is explained in Section V and the results of this study are described and discussed in Section VI. Section VII concludes this work and leads future research into several directions.

\section{RELATED WORK}

In a survey conducted by MacNamara et al. [8], 700 of 1015 participants thought printouts (voter verifiable audit trails) are important. However, a number of studies have shown that most voters do not verify the printouts: In the lab study conducted by Cohen [6] participants were asked to follow a voting agenda ${ }^{1}$, and were provided with instructions regarding the voting system in the voting booth. Only two of 36 participants detected any of the three types of manipulations (namely changing a vote to a different candidate, removing a vote from a candidate or a complete race) introduced in the printout. Further, Selker et al. [9] reports that in the "Arlington Voter Verification Study", only six of 35 participants verified the printouts. Thereby, participants were provided with instructions and verbal explanation regarding the voting system outside the voting booth, and the manipulations were similar to the one used by Cohen [6]. We acknowledge the problems identified by Whiteny et al. [10] regarding Selker et al.'s study design [9] which weakens the presented results. However, we mention it here as the trend not to verify printouts remains.

Other researchers report similar results but not based on reported detected manipulations in the printouts but based on their observation regarding the time participants spent with the verification step: Herrnson et al. [7] conducted a user study with more than 1400 participants. Before voting participants were asked to read a voting agenda. As a result of their study, authors state that most of the participants seem to ignore the printouts at all. The Center for American Politics and Citizenship conducted a similar user study in which they provided participants an overview of the verification mechanism, and in particular required them to pay attention to the verification step. They also report that most of the 815 participants did not spend the necessary time to verify all selections [5]. All these results motivate our own research.

\section{BACKGROUND}

We outline the general concepts and functionality of the electronic voting systems we address in this work by describing the system proposed by Volkamer et al. [4]. Many other electronic voting systems, for example [1], [2], [3] and [11] etc., are based on the same general concepts.

\footnotetext{
${ }^{1} \mathrm{~A}$ voting agenda refers to a pre-defined list of specific candidate(s) or a specific party.
}

\section{A. Vote Casting Process}

In the vote casting process the voter first identifies herself to the poll workers, similar to traditional paper-based system. Afterwards, the voter enters the voting booth and uses the electronic voting device to prepare the printout (ballot) by selecting candidates. When the voter confirms the selected candidates, the electronic voting device starts the printing process. The printout which contains the selections of the voter consists of two parts: a human-readable and a machinereadable part, namely a QR-Code. The QR-Code contains the exact information as the human-readable part, and it serves and enables an automatic tallying of printouts. The voter verifies that the human-readable part contains the candidates selected on the voting device. Finally, the voter folds the printout, leaves the voting booth and deposits the printout into the ballot box. The vote casting process is shown in Figure 1.

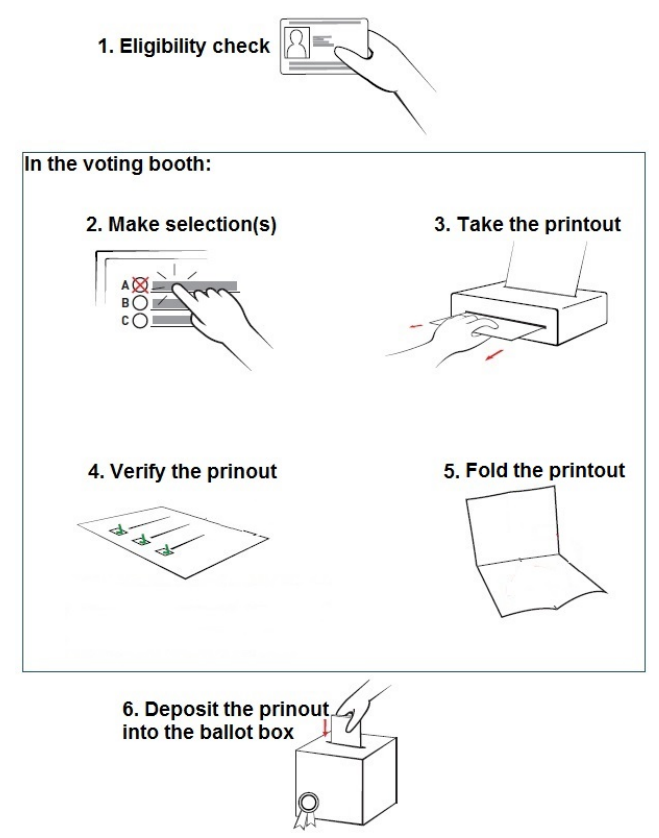

Fig. 1. Steps in the vote casting process.

\section{B. Tallying Process}

In the tallying process poll workers, where similar to the paper-based system the four-eyes principle is deployed, first open the sealed ballot box, and verify that the number of printouts (ballots) matches the number of voters. Afterwards, the poll workers start counting, by scanning the printouts one by one. Thus, they scan the QR-Code and verify that its content, shown on a monitor, matches the human-readable part of the printout. After the poll workers verify and confirm that the content on the human-readable part matches the content on the QR-Code, the scanned printout is added to the intermediate result, shown on a second monitor. The tallying process, for scanning/counting the printouts is shown in Figure 2.

As the election result is based on counting/auditing the printouts, it is very important that voters verify the content of the printouts, in order to detect a malicious voting device and ensure vote integrity and the integrity of the election result. 
1. Pick a ballot

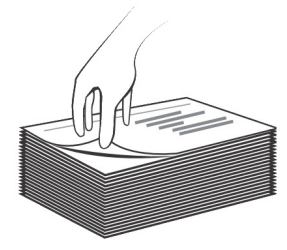

3. Compare Ballot with both monitors
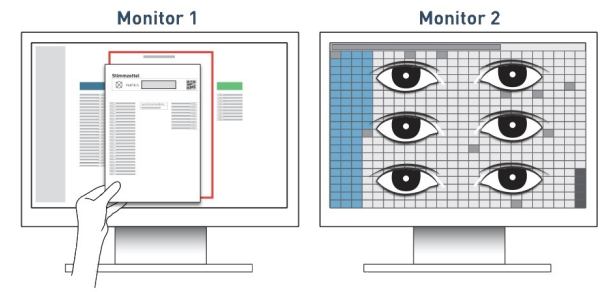

Fig. 2. Steps in the tallying process.

\section{Stimulus: Instructions, Position, Timing}

In this section, we describe the methodology for developing an adequate stimulus to temporarily divert voter attention from their main task (casting the printout) to verifying their printout.

\section{A. Instructions}

The instruction design is based on established research result: pictures and short sentences are used for better reading comprehension [12], and keywords are underlined and set in bold to improve information cognition [13]. Furthermore, instructions were set in a logical order and written in plain language, based on the guidelines recommended in [14], [15], and [16]. During pre-tests the instructions were iteratively improved, leading to a design consisting of two pictures accompanied by two main sentences (one for verifying and one for folding) and one auxiliary sentence, see Figure $3 .^{2}$ No word were emphasized in the auxiliary sentence, in order to not weaken the effect of the two main sentences.

Note that even though our focus lies on integrity of the vote, we included the second step after printing for controlling that the instructions do not cause that voters forget to fold their printout and thereby, accidentally, violate vote secrecy.

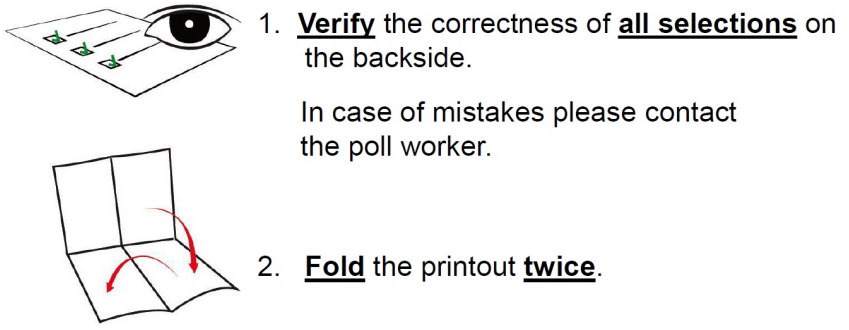

Fig. 3. Instructions for verifying the printouts (English translation).

\section{B. Position and Timing}

According to Nielsen?s [17], "Instructions [..] should be visible or easily retrievable whenever appropriate". In the

\footnotetext{
${ }^{2}$ The pictures were developed with the help of an external designer.
}

context of electronic voting Redish et al. [15] recommend that "Instructions must come "just in time" - when voters need them". This indicates that instructions for verifying the printouts should be provided during the vote casting process (and not only included in the election material). Displaying the instructions on the DRE screen, e.g. when the printing process start is inappropriate: a malicious DRE might not only manipulate the printout, but could also display incorrect instructions, e.g. "Thank you for voting. Deposit the printout into the ballot box.". Similar problems might occur if the instructions are printed by the DRE on the printout. Thus, we needed to provide the instructions without relying on the trustworthiness of the DRE and/or of the printer.

In order to identify the appropriate position and time we conducted a number of pre-tests with different approaches: posters on the screen, posters on the printer, front of the printout and reverse of the printout. We observed the behavior of participants during the pre-tests and carried out some informal interviews afterwards. Based on these results, the most promising approach, followed up in the user study, was participants (voters) first seeing the instructions on the printout, then turning the printout in order to verify its content. This had a positively surprising effect. The integration of the instructions in the vote casting process is shown in Figure 4 and animated in a video, which can be watched under the following link: http://tinyurl.com/oa94caj.

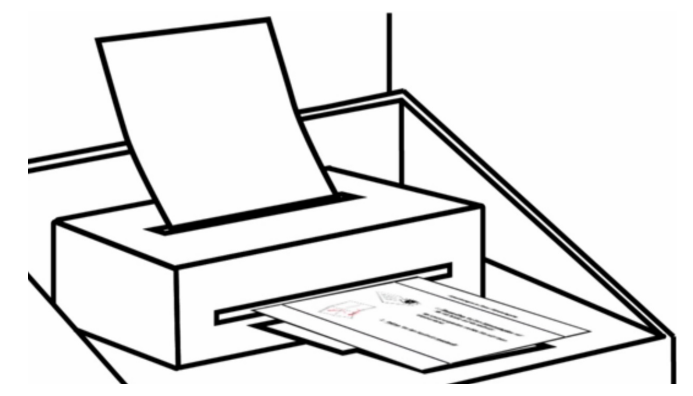

Fig. 4. Pre-printed instructions and their integration.

Note that the pre-prints are trusted because it is assumed that the poll workers insert them into the printer during the election setup phase.

\section{Study Design}

In this section we first explain why a cover story is necessary for evaluating the proposed stimulus and what criteria the cover story needs to meet. Then, we describe the cover story, experimental design, procedures and setup of our user study. We also report about recruiting and sampling of participants.

\section{A. Preliminary Considerations}

The general challenge in the area of usable security is that you cannot tell participants the primary goal of the study without biasing them [18]. Knowing the purpose of the study changes participants' behavior, such that they may act in a manner perceived as appropriate [19]. Thus, in our study participants might verify the printout (study goal), while this does not imply that they do so in a real election. In order to avoid such an effect, the communicated goal of the user study 
cannot be about the verification of the printout. Therefore, an appropriate method has to be considered for deceiving participants regarding the goal of our user study.

In addition, reliable results cannot be achieved through self-reported answers, and instead will require manipulation of the printouts. Due to legal and ethical concerns around manipulation, the study cannot be conducted during a legally binding election. An alternative might be to simulate an election. However, in an election simulation voters are likely to select those candidates they would also select in a real election which might lead to violation of vote secrecy. Thus, if a participant identifies the manipulation, and reveals the name of the selected candidate that was replaced (due to manipulating the printout), we would violate vote secrecy. Therefore, the user study cannot simulate an election. Last but not least, participants could be provided with a voting agenda, but then the printout has no personal relevance to them (compared to their vote in an election). ${ }^{3}$ Thus, the simulation of an election with a voting agenda, is also not appropriate for the research question we address in this work, because the printout must have a personal relevance for the participants. Asking participants personal, sensitive information would introduce a personal relevance to the printout. But, similar to election simulation, we would violate participant's privacy, if a participant identifies the manipulation, and reveals the information that was replaced (due to manipulating the printout). Thus, asking participants personal, sensitive information cannot be considered. Based on these requirements, we developed the following cover story, as the appropriate method for deceiving participants.

We are aware that related works, e.g. [5], [6] and [7], have simulated elections to evaluate whether voters verify or not the printouts (in terms of voter verifiable paper audit trails). However, as outlined in this section, an election simulation has more disadvantages with respect to evaluating the stimulus than the approach presented in this work.

\section{B. Cover Story}

The cover story we used in our user study is the following: The participants were told that they are taking part in a memory study which tests what type of information (e.g. numbers or names) people can better remember after having read a short text. After the reading task, participants were asked to give their answers on a computer, which provided them with a printout. Participants were told that evaluation is based on the answers on the printout they provide to the experimenter.

This cover story meets all requirements identified in the previous section. The primary goal of our study is completely hidden to the participants to avoid any effect caused by such knowledge. The stimulus is not evaluated within a voting scenario or by asking participants for personal, sensitive information. The printout can be manipulated without any concerns. Furthermore, the cover story ensures the following similar criteria regarding elections (1) Selections are made on an electronic device and printed. (2) Participants were told

\footnotetext{
${ }^{3}$ Note that we focus on the German federal elections, where voting is not compulsory. Therefore, we postulate that cast votes have a personal relevance for voters. However, as elections differ from country to country and some are compulsory, this might negatively influence voters' personal relevance with respect to their cast vote. Hence, this needs to be considered when designing user studies.
}

that they will be evaluated according to the results on their respective printout, thus introducing a personal relevance to the printout.

\section{Experimental Design and Procedure}

The study consisted of two groups: The control group got instructions as part of the experiment guidelines, simulating voters getting instructions for verifying the printout in the election material. The study group got the same instructions, but as part of the printout, simulating voters getting the instructions during the vote casting process.

The main goal of the user study is to analyze the effect of the stimulus on participants' awareness of and performance of verifying. In addition, we observed whether participants folded their printout. Correspondingly, the dependent variables were "awareness", "detection" and "folded". To measure "awareness" a post-questionnaire was developed. To measure "detection" the printout was manipulated and the study supervisor noted whether participants reported a problem. He also noted the state of printout when handed over to measure "folded". The independent variable consisted of the position and time of the instructions.

Each participant had to pass through eight sequential phases: First, each participant was provided with written experiment guidelines. The guidelines explained the cover story and the procedure of the experiment. In the second phase participants received a short (160 words) text about Alan Turing. Participants had three minutes to read and memorize the text. They were not allowed to take notes. Afterwards, participants had unlimited time to answer questions on the computer. For each of the 15 questions, three possible answers were provided. ${ }^{4}$ The participants had to answer all questions in order to proceed to printing. After participants confirmed the review screen, printing was started and the computer screen only displayed "Printing...". The selected answers were no longer displayed, similar to the process in the voting systems we consider in this work. Thus, after printing and by following the instructions (on the printout or in the election material), participants should verify their answers on the printout from memory. In case participants detected and reported the manipulation, the study supervisor asked about the type of problem to make sure it was about the error we introduced in the printout. After the participant explained the specific error, the study supervisor told the participant that he would "take note of the problem" and participants were encouraged to continue with the procedure in order to collect uniform and complete data. While participants handed the printouts to the experimenter, he took a note whether a participant folded the printout. In the next phase, the participants filled in the post-questionnaire. At the end of the experiment participants were debriefed and asked to not talk about the experiment with other persons, in order to avoid biasing other potential participants.

\section{Experimental Setup}

The setup used in the experiment is shown in Figure 5. Thereby, participants answered the questions on a computer, which logged all final answers. The quiz application was

\footnotetext{
${ }^{4}$ We included 15 questions, because this was the approximate number of parties in the last federal election in Germany.
} 
programed in Visual Basic for Applications for PowerPoint 2010. The instructions for verifying the printout, refer to Figure 3 in Section IV, were adapted to the cover story by replacing "all selections" with "all answers" and "poll worker" with "study supervisor". Participants of the control group were confronted with the blank side of the printout. This excludes turning of the printout as the cause for verifying its content in the study group.

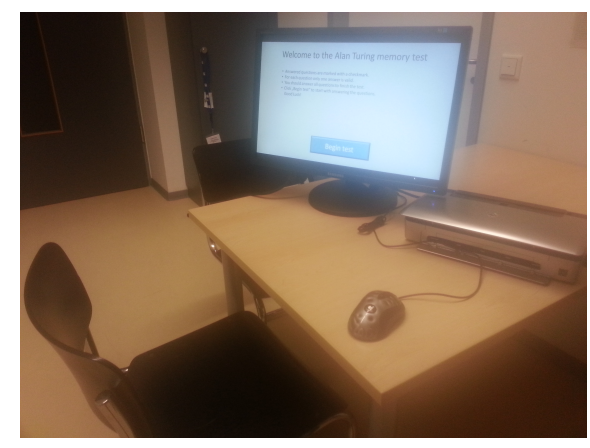

Fig. 5. Setting in the experiment.

\section{E. Manipulation}

We decided to manipulate the answer in the middle of the printout, as people in general are more likely to verify the first and the last items. Furthermore, we wanted to introduce a manipulation that is easy to detect, similar to changing the name of a candidate. Question seven "When was Alan Truing born?" seemed adequate regarding these requirements. The provided answers were 1910, 1911 and 1912, and independent from the answer given by the participant on the computer, 1845 was printed. We used 1845 for manipulating the printout, e.g. instead of 1921, because changing a candidate name implies changing at least a couple of characters.

\section{F. Post-questionnaire}

The post-questionnaire measured the following two questions using a five-point Likert scale anchored in "strongly disagree" and "strongly agree": (1) "I was aware that I should verify the correctness of all answers on the printout." (in order to evaluate the participants' awareness) (2) "I have verified the correctness of all answers on the printout." (in order to compare self-reported answers with reactions). In addition, the questionnaire collected some demographic data (sex, age, and background).

\section{G. Ethical Considerations}

An ethics commission at our university provides ethical requirements for research involving humans. These requirements were met; all participants read and confirmed the experimental guidelines, which stated that all data would be stored anonymously and serve only for the purpose of the experiment.

\section{H. Recruiting and Sample}

The participants were recruited via E-Mail and by personal contact. In the experiment participated 65 subjects ( 34 female, 41 male) at the age of 19-58 years. The sample consists of
40 students from different subjects, including psychologists, computer scientists, mechanical engineers, political scientists and architects; as well as 25 employees. The employees are classified as follows: academics of different levels (e.g. Dip., M.Sc., PhD) of their career, civil servants, freelancers, administrative technical staff members, caretakers, and event managers.

In order to control the effect of the stimulus, we decided to run an enhanced study group, i.e. to have more participants in the study group. For the study participants were randomly divided into two groups. In the study-group, we had 39 participants ( 20 female, 19 male) between 19-54 years, thereof 26 students and 13 employees. While in the control group, we had 26 participants (14 female, 12 male) between 2158 years, thereof 14 students and 12 employees. The size of the control group with 26 participants consists of a sufficient number of participants allowing robust statistical test to be carried out according to Lazar et al. [20]. Furthermore, we had two different compensations for the participants for motivating them to take part in our study. The psychology students are required from their department to participate in user studies for a specific amount of hours. Thus, in cooperation with the psychology department we compensated them with the appropriate amount of credits regarding our study. For the rest of the participants we provided a $20 €$ Amazon voucher. The voucher was randomly assigned to one of the participants from the group of participants that answered all questions correctly.

Note that all participants were naive, in scientific matter, towards the study. Furthermore, participants were only used to the paper-based voting system. Electronic voting systems were only very rarely used in Germany and only until 2005. The use of electronic voting systems in Germany for parliamentary elections has stopped with the introduction of the principle of "public nature of elections" by the German Federal Constitutional Court in 2009 [21], as the systems used until 2005 do not comply with this principle. In addition the experimenter was not known to research on security or electronic voting.

\section{RESULT AND DISCUSSION}

In order to measure the effect of the stimulus we evaluated the dependable variables, namely "detection", "folded" and "awareness". Participants of the study group detected the manipulation more frequently with $77 \%$ compared to $19 \%$ of participants from the control group. This includes all participants who detected and reported the manipulation to the study supervisor from the "voting booth", and continued with the procedure. In both groups all participants who detected the manipulation had selected the correct answer (at the quiz application). The percentage of people verifying based on instructions provided in the election material (i.e. in the experimental guidelines) is similarly low to those reported in early research. Note that in an real election scenario the number of people verifying the printout might be higher for both groups.

The evaluation of the second dependable variable shows that $90 \%$ of the study group participants folded the printout, in contrast to $27 \%$ of participants from the control group. This result indicates that the instructions did not distract participants from folding the printout. Further, we calculated a chi-square 
test over the dependable variables "detection" and "folded" to test their empirical relevance. For the variable "detection" the chi-quadrat-test returned a value of $\chi^{2}=(1, \mathrm{~N}=65)=20.89$ and a $\mathrm{p}$-Value of $\mathrm{p}<0.01$. Similarly, for the variable "folded" the chi-quadrat-test returned a value of $\chi^{2}=(1, \mathrm{~N}=65)=26.93, \mathrm{p}<$ 0.01 . This results in a highly significant difference between both groups.

Additionally, we compared the mean of the answers to the awareness question for both groups. The descriptive statistics show that the study group was more aware of verifying the printout (control group $\bar{x}=4.038$; study group $\bar{x}=4.837$ ). A Mann-Whitney significance test $(U(330.5) p<0.006))$ over the variable "awareness" was computed and showed that both groups differ significantly on how aware the participants were that they should verify the printout. Furthermore, a Pearson test shows that no correlation exists between verifying the printout age, education and gender respectively. We also evaluated the self-reported answers for both groups and compared them with the actual participants reaction, i.e. whether they actually detected the manipulation. In total 30 participants did not detect and report the error in the printout. However, all of them stated in the post-questionnaire to have verified the printout. This shows that it is necessary to trick participants as the self-reported statements are not reliable. Finally, we evaluated whether the different compensations affected the dependable variable "detection". The $\chi^{2}=(1, \mathrm{~N}=65)=2.52, \mathrm{p}>0.188$ shows that there is no significant difference between both groups, namely the psychology students and all other participants.

The results of our study show that all dependent variables "detection", "folded" and "awareness" are significantly higher in the study group than in the control group. This indicates that the proposed stimulus is a promising solution towards improving precautionary behavior in verifiable electronic voting systems.

\section{CONCLUSION AND FUTURE WORK}

There is no doubt that by using current electronic voting systems, election integrity is only ensured, if voters verify their printouts. Current research, for example [5] and [7], show that voters are not very likely to do so. This is a crucial issue even in countries with established democracy, like the U.S., Germany, U.K. etc. Therefore, it is very important to understand why voters are not very likely to verify their printouts and to motivate them to do so (of course without scaring them). Thus, in this work we developed an adequate stimulus to temporarily divert voter attention from their main task (putting the printout into the ballot box) to verifying their printout. The results of our work show that the proposed stimulus which is displayed and pre-printed on the printout (voter verifiable paper audit trail) is a promising solution towards motivating voters to verify.

\section{ACKNOWLEDGMENT}

This paper has been developed within the project 'VerkonWa' - Verfassungskonforme Umsetzung von elektronischen Wahlen - which is funded by the Deutsche Forschungsgemeinschaft (DFG, German Science Foundation). We would like to thank Paul Gerber for helpful comments and suggestions.

\section{REFERENCES}

[1] Board of Elections City of New York, Ballot Marking Device, Board of Elections City of New York Std., http://www.votethenewwayny.com/ en/using-the-new-voting-system.

[2] J. Ben-Nun, N. Fahri, M. Llewellyn, B. Riva, R. Rosen, Alon, A. T.-S Ta-Shma, and D. Wikström, "A New Implementation of a Dual (Paper and Cryptographic) Voting System," in Electronic Voting, 2012, pp. 315-329.

[3] C. Vegas, "The New Belgian E-voting System," in Electronic Voting, 2012, pp. 199-211.

[4] M. Volkamer, J. Budurushi, and D. Demirel, "Vote casting device with VV-SV-PAT for elections with complicated ballot papers," in International Workshop on Requirements Engineering for Electronic Voting Systems. Proceedings of the IEEE, 2011, pp. 1-8.

[5] "A Study of Vote Verification Technology Conducted for the Maryland State Board of Elections Part II: Usability Study," 2006, http:// www.capc.umd.edu/rpts/MarylandReport\%202-13-06.pdf (last accessed 18.09.2013).

[6] S. B. Cohen, "Auditing Technology for Electronic Voting Machines," 2005, master thesis, MIT, Media Lab.

[7] P. S. Herrnson, R. G. Niemi, M. J. Hanmer, P. L. Francia, B. B. Bederson, F. Conrad, and M. Traugott, "The promise and pitfalls of electronic voting: results from a usability field test," 2005, http://www. capc.umd.edu/rpts/Promise_and_Pitfalls_of_Electronic_Voting.pdf (last accessed 18.09.2013).

[8] D. MacNamara, T. Scully, J. Gibson, F. Carmody, K. Oakley, and E. Quane, "DualVote: Addressing Usability and Verifiability Issues in Electronic Voting Systems," in CeDEM11. Edition Donau-Universität Krems, 2011, pp. 313-322.

[9] T. Selker and A. Pandolfo, "A methodology for testing voting systems," Journal of Usability Studies, vol. 2, no. 1, pp. 7-21, 2006.

[10] Q. Whitney, C. John, C. Dana, K. Bill, and R. Ginny, "Comments on: Selker, Rosenzweig, and Pandolfo (2006). "A Methodology for Testing Voting Systems," JUS, Volume 2, Issue 1, 7-21," Journal of Usability Studies, vol. 2, no. 2, pp. 96-98, 2006.

[11] MSA Business Group, Vot.ar System, MSA Business Group Std., http: //www.vot-ar.com.ar/en/system-votation.

[12] D. Filippatou and P. D. Pumfrey, "Pictures, Titles, Reading Accuracy and Reading Comprehension: a research review (1973-95)," Educational Research, vol. 38, no. 3, pp. 259-291, 1996.

[13] S. J. Margolin, "Can Bold Typeface Improve Readers Comprehension and Metacomprehension of Negation?" Reading Psychology, vol. 34, no. 1, pp. 85-99, 2013.

[14] S. J. Laskowski and J. Redish, "Making ballot language understandable to voters," in Proceedings of the USENIX/Accurate Electronic Voting Technology Workshop 2006 on Electronic Voting Technology Workshop. USENIX Association, 2006, pp. 1-1.

[15] J. G. Redish and S. J. Laskowski, "Guidelines for Writing Clear Instructions and Messages for Voters and Poll Workers," NIST, Tech. Rep. 7596, 2009.

[16] American Institute of Graphic Arts, TOP 10 ELECTION DESIGN GUIDELINES, American Institute of Graphic Arts Std., http://www. aiga.org/election-design-top-ten/.

[17] J. Nielsen, "Heuristic Evaluation," in Usability Inspection Methods. John Wiley and Sons, 1994.

[18] C. Kuo, A. Perrig, and J. Walker, "Designing an evaluation method for security user interfaces: lessons from studying secure wireless network configuration," Interactions, no. 3, pp. 28-31, 2006.

[19] A. Sotirakopoulos, K. Hawkey, and K. Beznosov, “'I did it because I trusted you": Challenges with the Study Environment Biasing Participant Behaviours," in SOUPS, 2010.

[20] J. Lazar, J. Feng, and H. Hochheiser, Research Methods in HumanComputer Interaction. Wiley, 2010.

[21] Federal Constitutional Court of Germany, Federal Constitutional Court of Germany: Entscheidungen des Bundesverfassungsgerichts(BVerfGE), BVerfGE 123, 39 - 88, Federal Constitutional Court of Germany Std., March 2009, http://www.bverfg.de/entscheidungen/rs20090303_ 2bvc000307en.html. 